\title{
Enhancement of wound healing by single-wall/ multi-wall carbon nanotubes complexed with chitosan
}

This article was published in the following Dove Press journal:

International Journal of Nanomedicine

\section{Naim Kittana' \\ Mohyeddin Assali ${ }^{2}$ \\ Hanood Abu-Rass' \\ Susanne Lutz ${ }^{3,4}$ \\ Rama Hindawi ${ }^{2}$ \\ Lina Ghannam ${ }^{2}$ \\ Marah Zakarneh² \\ Ahmad Mousa'}

'Department of Biomedical Sciences, Faculty of Medicine and Health Sciences, An-Najah National University, Nablus, Palestine; ${ }^{2}$ Department of Pharmacy, Faculty of Medicine and Health Sciences, An-Najah National University, Nablus, Palestine; ${ }^{3}$ Institute of Pharmacology and Toxicology, University Medical Center, Göttingen, Germany; ${ }^{4}$ German Center for Cardiovascular Research e.V., Partner Site, Göttingen, Germany
Correspondence: Naim Kittana

Department of Biomedical Sciences, Faculty of Medicine and Health Sciences, An-Najah National University, Akademia Street, PO Box 7, Nablus, Palestine

$\mathrm{Tel}+97092345 \mathrm{II} 3$

Fax+970 92345982

Email naim.kittana@najah.edu
Background: Impaired wound healing is commonly associated with many health problems, including diabetes, bedsores and extensive burns. In such cases, healing often takes a long time, which subjects patients to various complications. This study aims to investigate whether single-wall or multi-wall carbon nanotubes complexed with chitosan hydrogel can improve wound healing.

Materials and methods: Initially, the effects of the complexes on the viability and functionality of fibroblasts were investigated in engineered connective tissues. Then, their activity on wound healing was investigated in a mouse model with induced full-thickness wounds, in which the wounds were treated daily with these complexes. Finally, the effect of the complexes on collagen deposition by fibroblasts was investigated in vitro.

Results: The engineered connective tissue studies showed that fibroblasts were viable in the presence of the complexes and were still able to effectively organize and contract the extracellular matrix. In vivo data showed that both types of complexes improved the re-epithelialization of the healing wounds; however, they also increased the percentage of wounds with higher fibrosis. In particular, the chitosan-multi-wall carbon nanotube complex significantly enhanced the extensiveness of this fibrosis, which is in line with in vitro data showing a concentrationdependent enhancement of collage deposition by these complexes. These observations were associated with an increase in inflammatory signs in the wound bed.

Conclusion: Single-wall and multi-wall carbon nanotubes complexed with chitosan improved the re-epithelialization of wounds, but an increase in fibrosis was detected.

Keywords: carbon nanotubes, chitosan, wound healing, re-epithelialization, fibrosis, inflammation

\section{Introduction}

The integumentary system consists of skin and its accessories, including hair follicles, sweat glands and sebaceous glands. It provides various indispensible protective and regulatory functions. Throughout a person's life, skin is repeatedly subjected to many kinds of injuries, which usually heal within a relatively short time by perfect regeneration or with various degrees of scarring. However, some skin injuries, such as severe burns, diabetic foot and bed ulcers may take several months to heal, if they ever do, which requires prolonged hospitalization and may predispose patients to serious infections and complications. ${ }^{1,2}$ The development of pharmaceutical preparations that can accelerate the healing process has long been the focus of many research groups and pharmaceutical companies worldwide. ${ }^{3}$ Among the many substances investigated for that purpose is a natural polymer called chitosan, which has the advantages of being non-toxic and 
biocompatible. Chitosan has been incorporated in various topical formulations including powders, hydrogels and dressings, and these preparations have been tested in wound and burn animal models. In general, chitosan-containing preparations can increase the rate of wound re-epithelialization, fibroblast proliferation, collagen deposition and the tensile strength of the healing wound. ${ }^{4}$ In addition, they exhibit good antimicrobial properties, which is important for wound care. ${ }^{5}$ In vivo data on an open skin wound model showed that chitosan-based preparations improved re-epithelialization, enhanced the production of collagen by fibroblasts and accelerated the infiltration of polymorphonuclear cells in the wound bed. ${ }^{6,7}$ Clinical studies have reported that chitosan preparations improved the histoarchitectural order and vascularization of the dermal layer. ${ }^{8}$ It is possible to further enhance the activity of chitosan, possibly by conjugating it with macromolecules such as carbon nanotubes (CNTs) that improve the adhesion of chitosan to the wounded surface and may enhance the interaction of chitosan with cells in the wound bed.

CNTs are an allotropic form of carbon with dimensions in the nanoscale and length in the micrometer scale that form a needle-like structure with a large surface area. ${ }^{9,10}$ The carbon atoms bind to each other, forming sheets of six-membered carbon atom rings (graphite) that wrap into cylindrical tubes. ${ }^{11,12}$ Depending on the number of graphite layers, CNTs can be classified into single-wall carbon nanotubes (SWCNTs) and multiwall carbon nanotubes (MWCNTs). ${ }^{13}$ The tensile strength of CNTs can be up to 63 gigapascals ( $\mathrm{GPa}$ ), which is around 50 times greater than that of steel, ${ }^{14}$ while their elastic modulus can range between 1.0 and 1.8 terapascals (TPa).${ }^{15}$ In general, pristine CNTs (CNTs without the addition of any hydrophilic functional group) do not disperse in water and they tend to aggregate and precipitate in aqueous solutions. ${ }^{16,17}$ Pristine CNTs may produce several toxic reactions, but the addition of polar functional groups to pristine CNTs can significantly improve the dispersibility of CNTs in aqueous environments, and at the same time greatly reduce their toxicity. ${ }^{18-20} \mathrm{CNTs}$ are currently being functionalized by different kinds of pharmacologically active molecules with the aim of enhancing drug delivery, controlling drug release and improving drug activity, which has been successful to a certain extent. ${ }^{21,22}$

In this project, SWCNTs and MWCNTs were complexed with chitosan, which is a hydrophilic polymer, via van der Waals' interactions, so that the chitosan polymer would wrap on to the CNT with the hydrophilic regions of chitosan exposed to the aqueous environment. This resulted in a hydrogel incorporating a scaffold of CNTs (chitosan-single-wall carbon nanotubes [C-SWCNTs] and chitosan-multi-wall carbon nanotubes [C-MWCNTs]). Then, the effect of C-SWCNTs and C-MWCNTs on collagen deposition and extracellular matrix organization by fibroblasts was investigated. The effects of these compounds on the healing process were then studied in a mouse model with induced full-thickness wounds.

\section{Materials and methods Materials}

SWCNTs and MWCNTs were purchased from Carbon Solutions (Riverside, CA, USA). Acetic acid, chitosan, Sirius Red, picric acid, formaldehyde and tetramethylrhodamine (TRITC)-phalloidin were purchased from Sigma-Aldrich (St Louis, MO, USA). DMEM 1× solution with high glucose, DMEM powder with high glucose, FBS, penicillin/ streptomycin and PBS were purchased from Gibco (Waltham, MA, USA). Sterile water $\left(\right.$ Aqua $^{\circledR}$ ) was purchased from Braun (Kronberg im Taunus, Germany). Acid-soluble collagen was purchased from Devro (Chryston, UK). Roti ${ }^{\circledR}$-Block, Triton X-100 and Roti ${ }^{\circledR}$-Histofix 4\% were purchased from Carl Roth (Karlsruhe, Germany), DAPI was purchased from Hoffman-La Roche Ltd. (Basel, Switzerland). The 3 T3 fibroblast cell lines were a kind gift from Dr Johnny Amer (American Type Culture Collection [ATCC], Manassas, VA, USA) and human foreskin fibroblasts were obtained from ATCC.

\section{Preparation of C-SWCNT/C-MWCNT complexes}

To prepare solutions of C-SWCNT and C-MWCNT complexes, chitosan solution with a concentration of $0.5 \%$ was prepared in $0.5 \%$ acetic acid aqueous solution. After that, SWCNTs or MWCNTs were added and the mixtures were then sonicated for 2 hours.

For the preparation of C-SWCNTs and C-MWCNTs in the form of a hydrogel, first of all 1\% w/v chitosan solution was prepared in $1 \%$ aqueous acetic acid solution under sonication for 15 minutes. The solution was then used to prepare $1 \%$ and $5 \%$ mixtures of either SWCNTs or MWCNTs. The mixtures were sonicated for 30 minutes and then their $\mathrm{pH}$ was titrated to 7.34 using $1 \mathrm{~N} \mathrm{NaOH}$ solution. ${ }^{23}$ Then, the mixtures were centrifuged for 10 minutes at $15,000 \mathrm{rpm}$, and the resulting supernatant was removed and the separated hydrogel was washed twice with distilled water. Finally, polyethylene glycol (PEG) was added to the hydrogel as $5 \% \mathrm{v} / \mathrm{v}$ to improve wettability and to enhance the adherence of the hydrogel to the wound surface. Samples from the resulting hydrogels were sent to the University of Jordan for imaging with a transmission electron microscope (TEM). 


\section{Generation and processing of engineered connective tissues (ECTs)}

ECTs were generated according to the method described by Tiburcy et al for the preparation of engineered heart muscle, with the exception that in this project the type of cells incorporated was human foreskin fibroblasts. ${ }^{24}$ In brief, $10 \times$ DMEM was prepared from DMEM powder, from which $2 \times$ DMEM was prepared by diluting in Aqua water with the addition of serum up to $20 \%$. CNT solution was mixed with $2 \times$ DMEM, then acid-soluble collagen was added, followed by neutralization of the mixture with $0.1 \mathrm{~N} \mathrm{NaOH}$, after which the cell suspension was added so that each ECT would contain $1.4 \times 10^{6}$ cells. Up to this step, all processes were performed on ice. After this, the mixture was cast in silicon molds similar to those used by Tiburcy et al. ${ }^{24}$ The molds were kept in a cell culture incubator for 1 hour, after which they received high-glucose DMEM (containing 15\% serum and $1 \%$ penicillin/streptomycin). The tissues were kept in culture for 5 days before they were imaged using a Lumar.V12 ${ }^{\circledR}$ stereomicroscope (Zeiss). The tissues were then fixed in Roti-Histofix 4\% overnight, after which they were washed three times with PBS and once with $70 \%$ ethanol before they were embedded in $2 \%$ agarose gel and sectioned with a VT1000S Vibratome ${ }^{\circledR}$ (Leica). The tissue slices were permeabilized with $0.05 \%$ Triton for 10 minutes, blocked for 1 hour in Roti-Block solution, then stained overnight with TRITC-phalloidin and DAPI.

\section{In vivo experiments on wound healing Experimental animal set-up}

Twenty-five CC-72 line mice were obtained from the animal facility at An-Najah National University in Nablus, Palestine. The mice were randomly equally distributed into five groups, labeled: Chitosan, 1\% C-SWCNT, 5\% C-SWCNT, 1\% C-MWCNT and 5\% C-MWCNT. The mice were left in the cages for a few days to acclimatize, and had free access to water and feed throughout the experiment. The animal handling and the procedures were carried out according to the guidelines of the Ethics Committee of the International Association for the Study of Pain, and the experimental and handling protocol was further reviewed and approved by the institutional review board committee at An-Najah National University.

\section{Induction of wounds}

A commercial hair-removal cream, Nair (Church \& Dwight, Ewing Township, NJ, USA), was used to remove the fur from the dorsal surface of all mice. Under deep anesthesia with inhaled isoflurane, two wounds, one on either dorsal side, were induced in each animal by excising a circle of full skin thickness (diameter about $1 \mathrm{~cm}$ ). The left-side wound was left untreated to serve as an internal negative control, while the right-side wound received daily treatment with the assigned hydrogel over 9 days. After this, the mice were killed with excessive anesthesia, and autopsies were collected from the sites of the healing wounds, which were stored in formalin for further histopathological evaluation. ${ }^{3}$ All tissue samples were processed and sectioned according to the standard professional procedure followed routinely in the medical laboratory of An-Najah National University Hospital for regular pathological samples. The samples were stained with H\&E and Masson's trichrome stain, and then examined by a clinical pathologist who was blinded to the samples. In addition, the degree of fibrosis was digitally evaluated by analyzing the stained tissue images using Image $\mathrm{J}^{\circledR}$ software, as detailed below.

\section{Digital evaluation of degree of fibrosis and re-epithelialization}

Three representative microscopic images were taken for each sample stained with Masson's trichrome stain, using the $10 \times$ lens. Semi-quantification of the degree of fibrosis was performed digitally using ImageJ software according to a method published by Chen et al. ${ }^{25}$ The "color deconvolution" plugin was used to measure the integrated density for the green color channel (the product of mean gray value and area) for the tissue samples. ${ }^{25}$ Epidermal thickness was measured using the "straight line" tool in ImageJ. At least three measurements were taken for each sample at three different locations. The statistical analysis was conducted using the Student's $t$-test and graphs were obtained using GraphPad Prism $^{\circledR}$ software (GraphPad Software, La Jolla, CA, USA).

\section{In vitro investigation into the effect of C-SWCNT/C-MWCNT complexes on cell behavior and collagen deposition}

The $3 \mathrm{~T} 3$ fibroblast cell line was used as a model to investigate the effect of C-SWCNTs and C-MWCNTs on collagen deposition. The cells were evenly seeded in 12-well plates and maintained overnight in DMEM containing 10\% FBS. On the next day, the medium was removed and the cells were washed once with PBS, followed by incubation over 72 hours with fresh DMEM containing 2\% FBS in addition to various concentrations of either SWCNTs or MWCNTs. At the end of the incubation time, the cells were washed three times with PBS, followed by incubation for 1 hour at room temperature with Bouin's solution (composed of $71 \%$ saturated aqueous 
picric acid, 24\% formaldehyde-35\% and 5\% acetic acid). Next, Bouin's solution was removed and the plates were washed under running tap water for 15 minutes before they were incubated with $0.1 \% \mathrm{w} / \mathrm{v}$ Sirius Red stain solution (prepared in saturated aqueous picric acid) for 1 hour at room temperature with mild shaking. The dye was then removed and the cells were washed extensively with $0.01 \mathrm{~N} \mathrm{HCl}$ to remove the excess dye. Then, the wells were incubated for 30 minutes with $0.1 \mathrm{~N} \mathrm{NaOH}(0.5 \mathrm{~mL} /$ well $)$ under mild shaking to release the collagen-bound stain. Finally, the absorbance of the Sirius Red stain in the supernatant was measured at a wavelength of $550 \mathrm{~nm}$, which is the lambda $\max \left(\lambda_{\max }\right)$ for this stain. ${ }^{26}$ It is noteworthy that all of the CNTs were completely eliminated by the previous extensive washing steps, and were therefore not expected to interfere with the measured absorbance.

\section{Results}

\section{CNTs were able to complex with chitosan polymer in a hydrogel}

Samples of the tested hydrogels imaged by TEM demonstrated the successful formation of an interweaving network of CNTs with the chitosan polymer (Figure 1). The network was more prominent with 5\% C-MWCNTs (Figure 1D).

\section{C-SWCNTs and C-MWCNTs were compatible with connective tissues}

To investigate the compatibility of C-SWCNTs and C-MWCNTs in dermal-like connective tissues in vitro, these compounds were incorporated in the extracellular matrix of dermal ECTs. The tissues were sectioned and stained with TRITC-phalloidin to fluorescently stain the actin cytoskeleton, and DAPI was used to stain the nuclei so as to mark the fibroblasts within the tissues. As shown in the control tissues in Figure 2 (upper panel), the dermal fibroblasts were aligned along the axis of the tissue and they were able to organize and contract the matrix, forming a consistent connective tissue. In the presence of C-MWCNTs and C-SWCNTs, the fibroblasts were also able to form ECT, with the particles of the CNTs well scattered within the extracellular matrix, as shown in Figure 2 (middle and lower panels).

\section{CNTs improved wound healing in vivo}

The in vivo experiments were based on preparing a hydrogel composed of chitosan alone or chitosan complexed with either SWCNTs or MWCNTs, so as to give the CNTs hydrophilic properties that would allow effective contact with skin lesions and adequate dispersal in aqueous media.
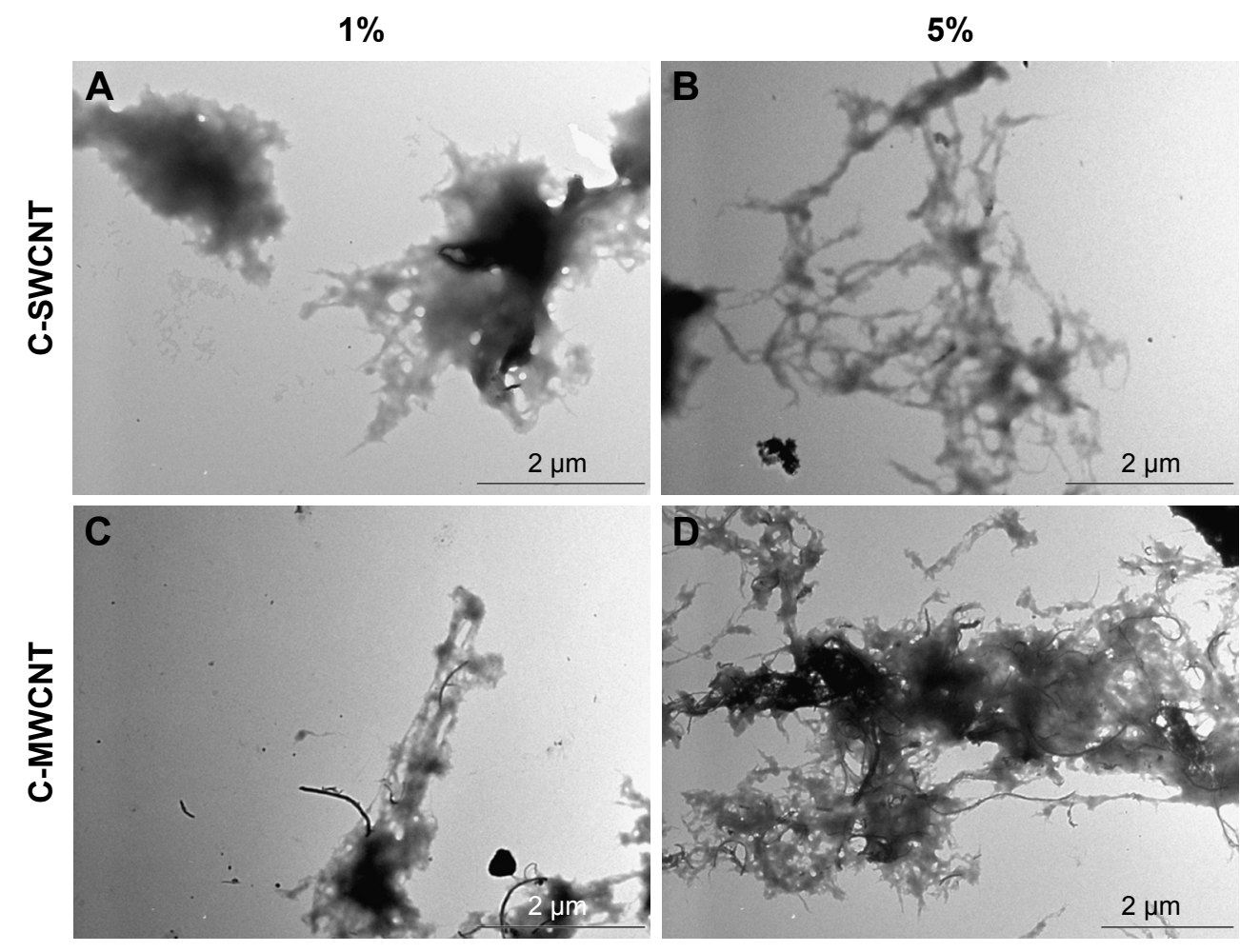

Figure I Formation of the carbon nanotube-chitosan complex.

Notes: Transmission electron microscope imaging demonstrated the formation of an interweaving network of chitosan polymer with carbon nanotubes. (A) I\% C-SWCNT; (B) $5 \%$ C-SWCNT; (C) 1\% C-MWCNT; (D) 5\% C-MWCNT.

Abbreviations: C-SWCNT, chitosan-single-wall carbon nanotube; C-MWCNT, chitosan-multi-wall carbon nanotube. 


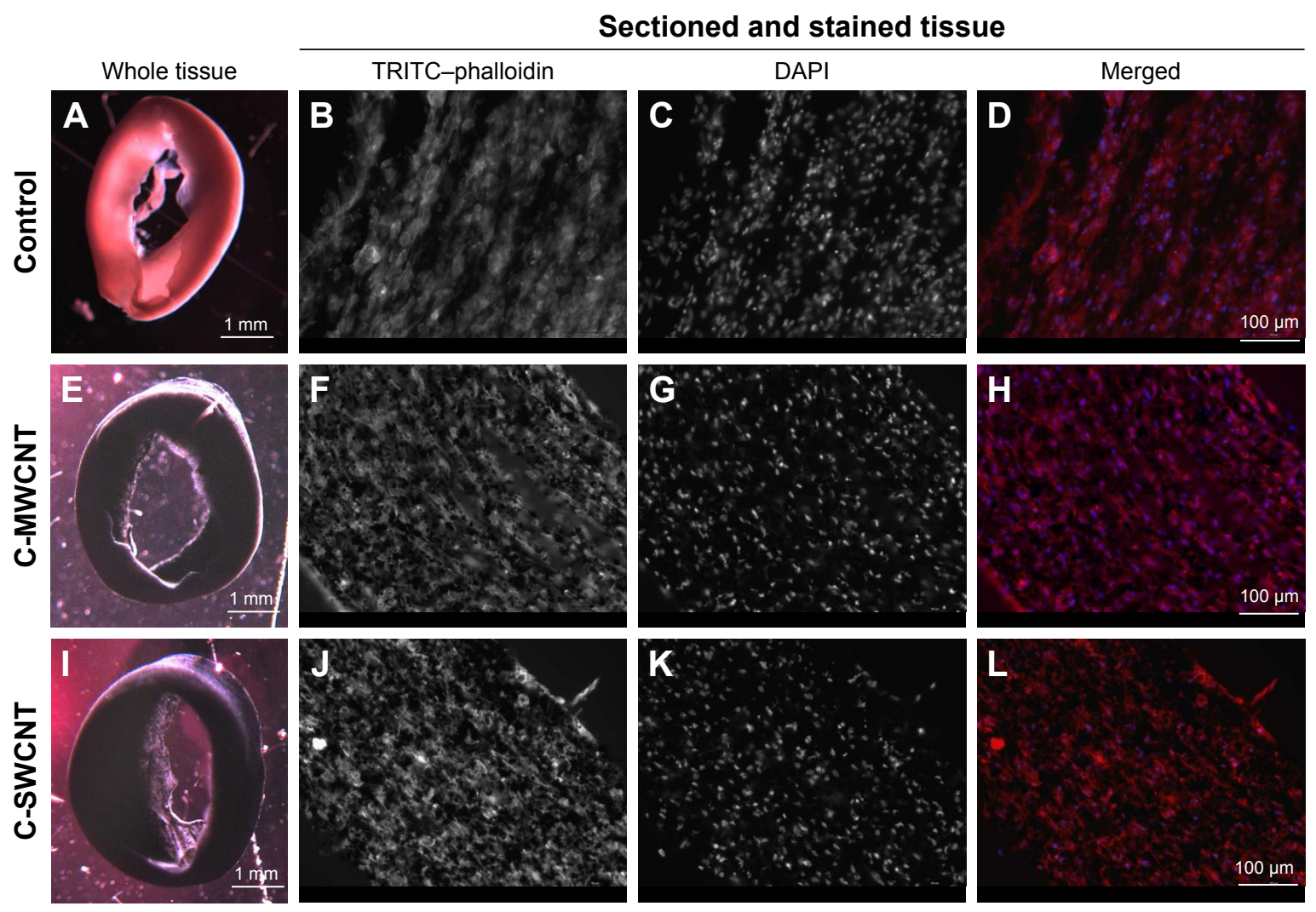

Figure 2 Fibroblasts formed ECT in vitro in the presence of C-SWCNTs and C-MWCNTs.

Notes: (A, E, I) Bright-field images for intact ECT; (B, F, J) actin cytoskeleton of the fibroblasts stained with TRITC-phalloidin (red); (C, G, K) nuclei stained with DAPI (blue); (D, H, L) merged images from DAPI and TRITC-phalloidin.

Abbreviations: ECT, engineered connective tissue; C-SWCNT, chitosan-single-wall carbon nanotube; C-MWCNT, chitosan-multi-wall carbon nanotube; TRITC, tetramethylrhodamine.

\section{Wounds treated with CNTs closed more quickly than controls}

After 9 days of treatment, most of the wounds were almost completely healed. Figure 3A, C, E, G and I demonstrate the healing of the internal controls for each of the treatment conditions. In comparison with these controls, the stage of the healing process was more advanced for wounds treated with 1\% C-SWCNTs and 5\% C-MWCNTs (Figure 3J and K), where the wounds were drier, better closed and smaller in size, compared to the other conditions (Figure 3B, D, F and $\mathrm{H})$. However, to evaluate the quality and efficiency of the healing process, the healing sites were excised after the mice had been killed, and were sent for histopathology.

\section{Clinical histopathological evaluation demonstrated an enhanced fibrotic process and increased inflammation by treatment with CNTs}

A blinded histopathological investigation of histology samples stained with Masson's trichrome stain demonstrated that $80 \%$ of the wounds treated with $1 \%$ C-SWCNTs or $5 \%$ C-MWCNTs and $60 \%$ of those treated with $5 \%$ C-SWCNTs had more fibrosis compared to the internal control wounds. However, only $40 \%$ of the wounds treated with chitosan or $1 \%$ C-MWCNTs had more fibrosis, as shown in the graph in Figure 4A. This was regardless of the intensity of fibrosis. At the same time, using H\&E stain it was found that wounds treated with any of the test hydrogels had more infiltration by inflammatory cells (with the exception of 1\% C-MWCNTs), as shown in the graph in Figure 4B, which may reflect higher inflammatory activity in these wounds.

\section{Digital analysis of the tissue slides demonstrated greater intensity of fibrosis with 5\% C-MWCNT treatment}

To evaluate the intensity of fibrosis under the test treatment conditions, tissue samples from the healing regions were stained with Masson's trichrome stain (Figure 5), and then digital analysis was performed for random images taken of the stained wound tissue slides (Figure 6). Statistical analysis of the data demonstrated significantly greater integrated density values for wounds treated with 5\% C-MWCNTs compared to the control wounds, which reflects the greater 


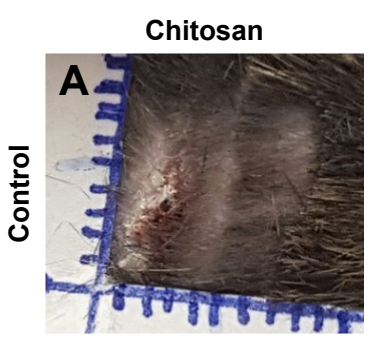

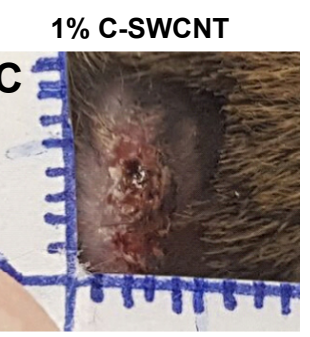

$5 \%$ C-SWCNT
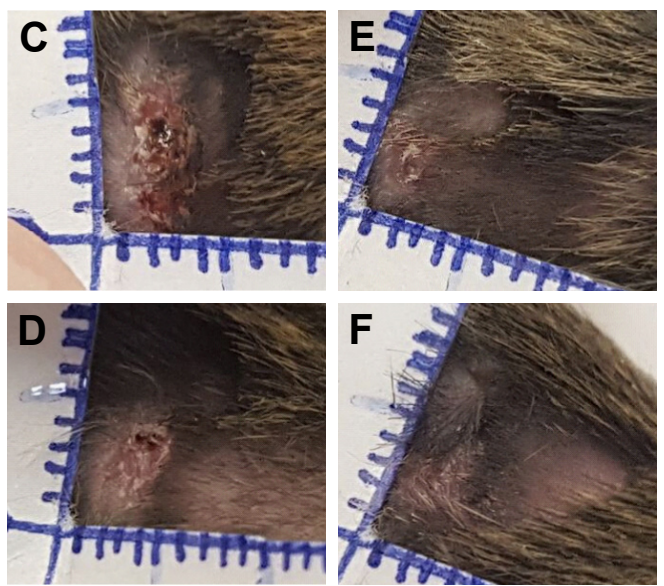

$1 \%$ C-MWCNT
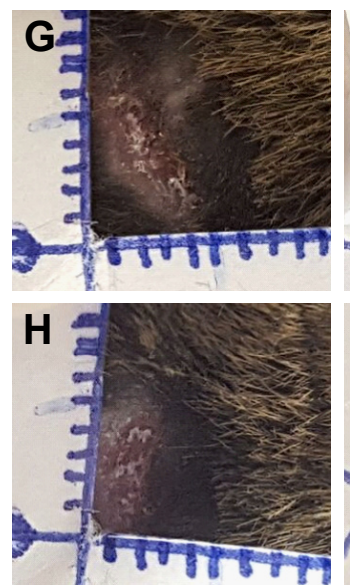

5\% C-MWCNT
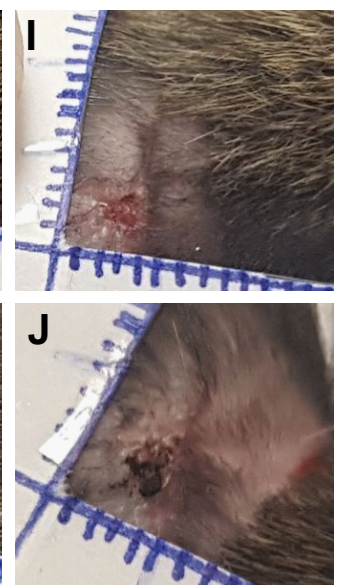

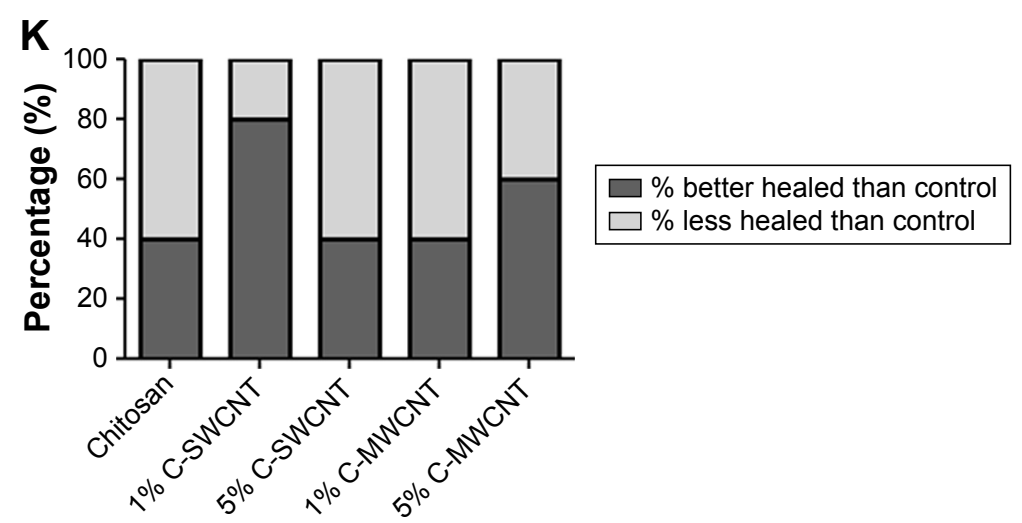

Figure 3 Macroscopic evaluation of the wounds at the end of the 9-day treatment course.

Notes: (A, C, E, G, I) Healing of the internal controls for each of the treatment conditions; (B) following treatment with chitosan; (D) following treatment with I\% C-SWCNT; (F) following treatment with 5\% C-SWCNT; (H) following treatment with 1\% C-MWCNT; (J) following treatment with $5 \%$ C-MWCNT; (K) clinical scoring for the healing wounds at the end of the treatment course with the different conditions. Wounds treated with I\% C-SWCNT and 5\% C-MWCNT hydrogel closed more rapidly than the corresponding internal controls. Each tick corresponds to I mm $(n=5)$.

Abbreviations: C-SWCNT, chitosan-single-wall carbon nanotube; C-MWCNT, chitosan-multi-wall carbon nanotube.
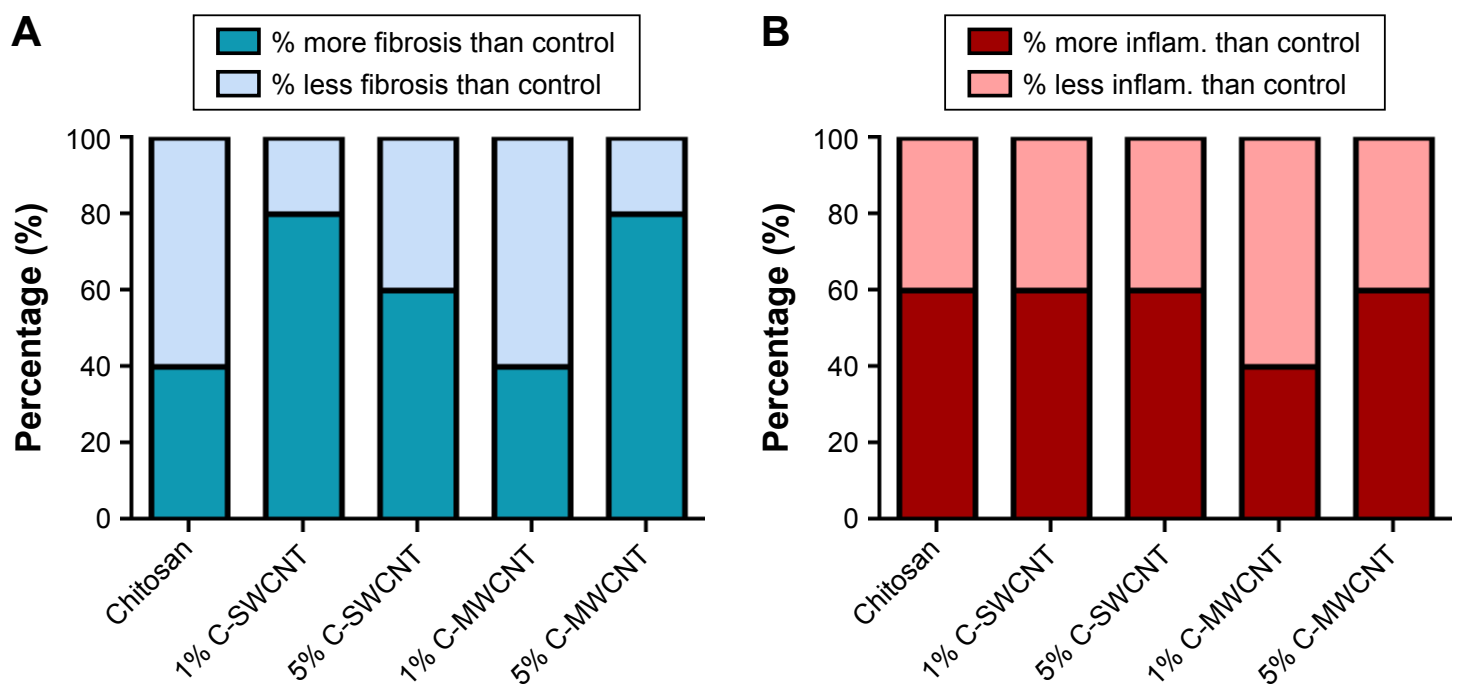

Figure 4 Treatment with 5\% C-SWCNTs and 5\% C-MWCNTs may increase the percentage of wounds with more fibrosis and more inflammation.

Notes: (A) Percentage of wounds with more/less fibrosis than the corresponding control; (B) percentage of wounds with more/less inflammation than the corresponding control $(n=5)$.

Abbreviations: C-SWCNT, chitosan-single-wall carbon nanotube; C-MWCNT, chitosan-multi-wall carbon nanotube; inflam., inflammation. 

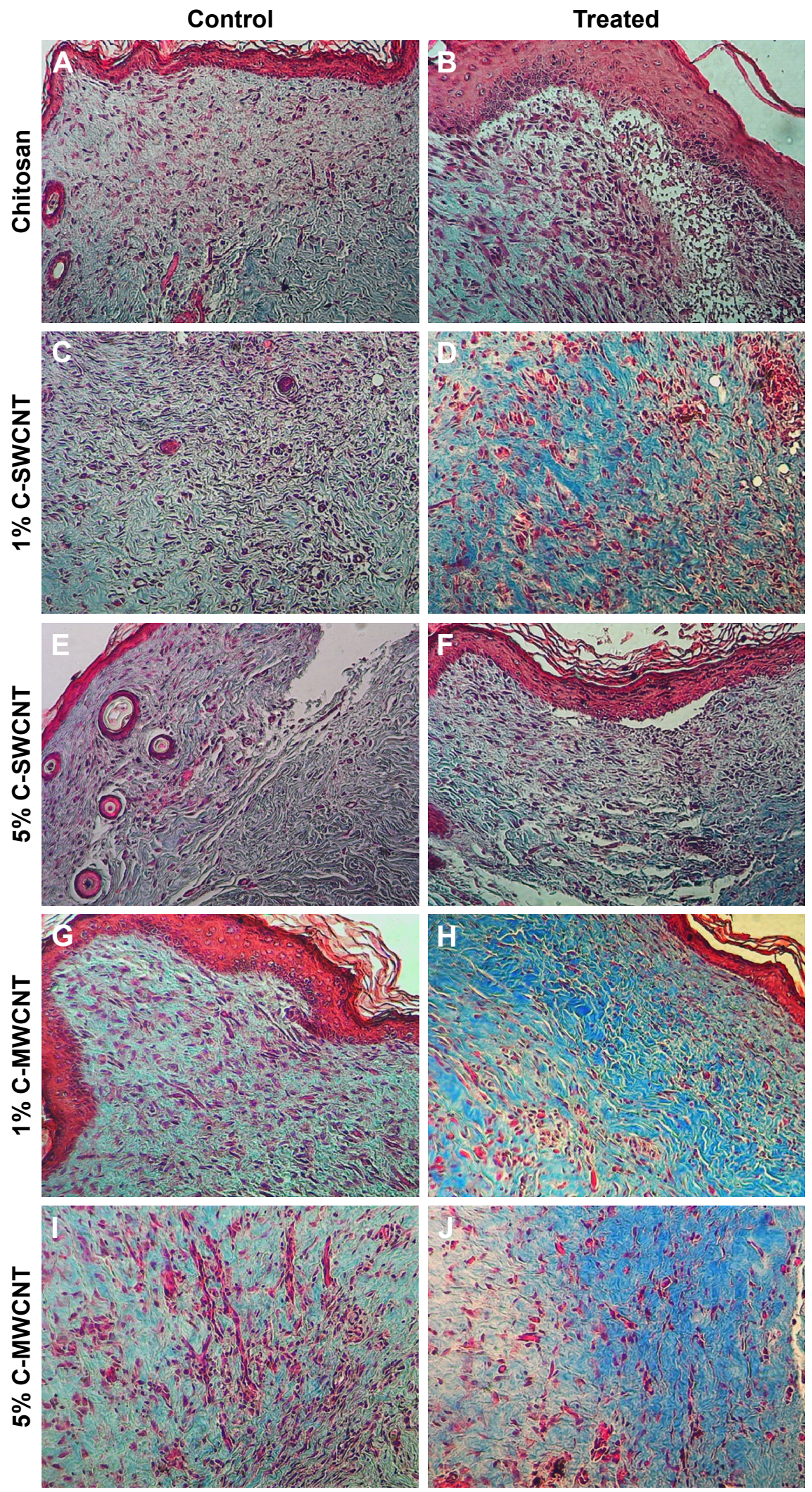

Figure 5 Treatment with 5\% C-MWCNTs best improved the fibrotic processes in healing wounds.

Notes: Masson's trichrome stain for sectioned full-thickness skin tissue samples collected from the site of the healing wounds at the end of the 9-day treatment course. The bluish-green color represents the stained collagen. Magnification 100×. (A, C, E, G, I) Internal controls of the corresponding treatment conditions; (B) following treatment with chitosan; (D) following treatment with I\% C-SWCNTs; (F) following treatment with 5\% C-SWCNTs; (H) following treatment with I\% C-MWCNTs; (J) following treatment with 5\% C-MWCNTs.

Abbreviations: C-SWCNT, chitosan-single-wall carbon nanotube; C-MWCNT, chitosan-multi-wall carbon nanotube. 

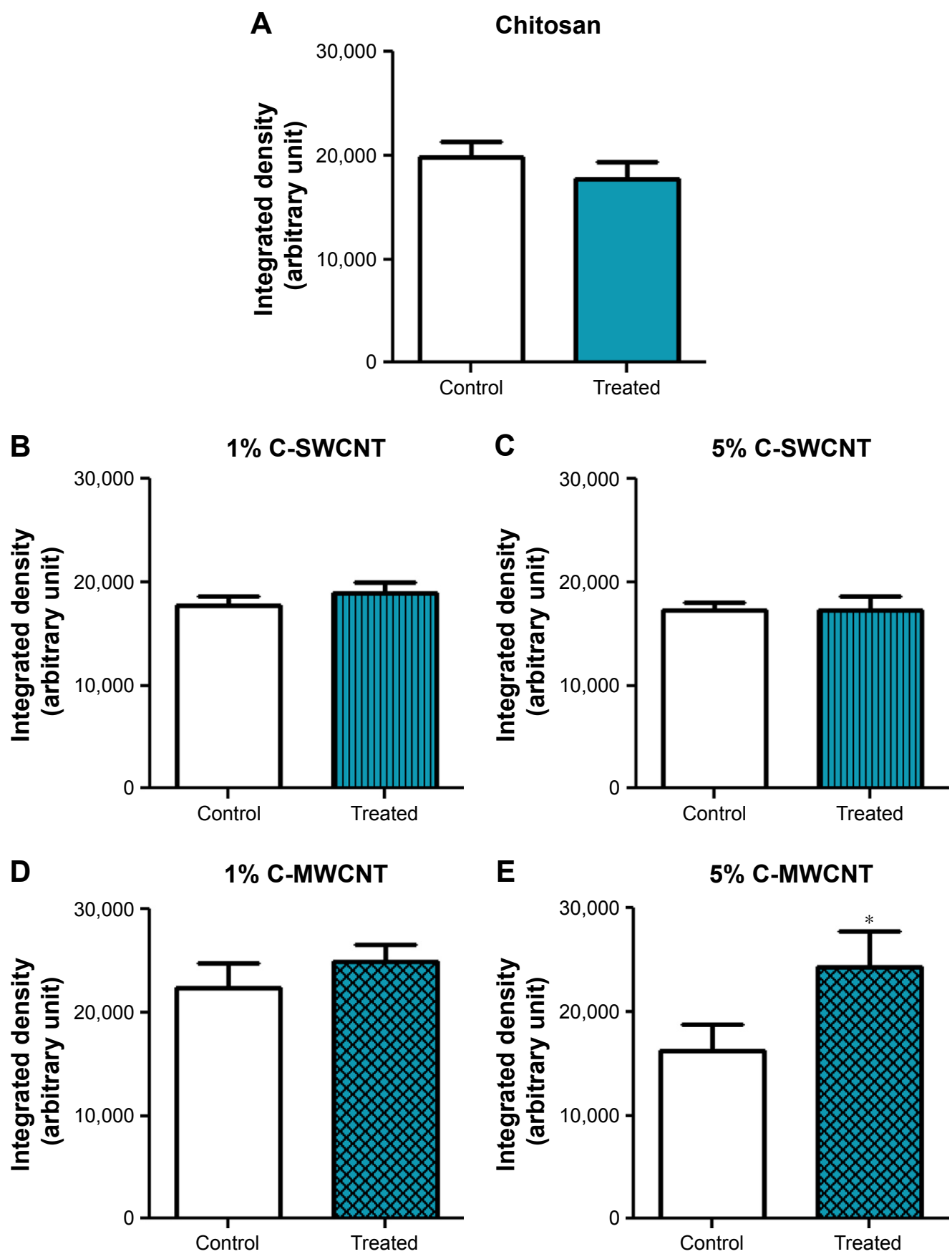

$\mathbf{E}$

$5 \%$ C-MWCNT

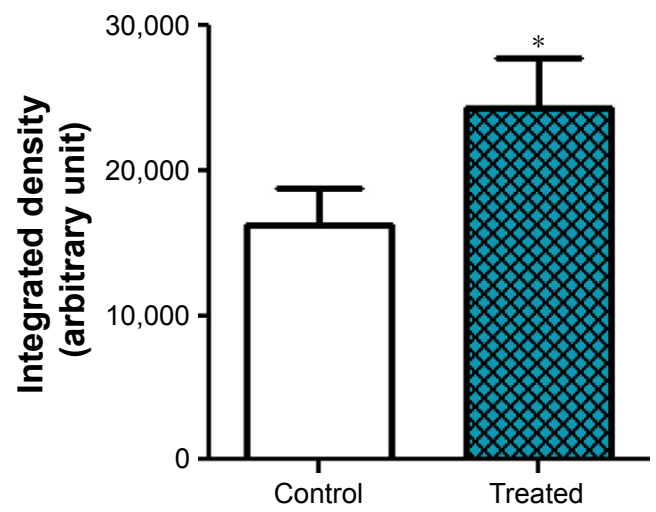

Figure 6 C-MWCNT was the most effective treatment to increase the amount of deposited collagen in the healing wound.

Notes: Images for tissue samples stained with Masson's trichrome stain were digitally analyzed by the "color deconvolution" plugin in Image). (A) Treatment with chitosan; (B) treatment with 1\% C-SWCNTs; (C) treatment with 5\% C-SWCNTs; (D) treatment with 1\% C-MWCNTs; (E) treatment with 5\% C-MWCNTs. Values are given as means \pm SEM, $n=5$, triplicates. $* P<0.05$ compared to the control. Treatment with $5 \%$ C-MWCNTs had a significantly greater effect than that of the corresponding internal control. For the other treatment conditions there was no significant difference.

Abbreviations: C-SWCNT, chitosan-single-wall carbon nanotube; C-MWCNT, chitosan-multi-wall carbon nanotube.

amount of deposited collagen in these samples, as shown in Figures $5 \mathrm{~J}$ and $6 \mathrm{E}$. Such an effect was not significant with $1 \%$ C-MWCNTs (Figures 5H and 6D). Treatment with chitosan, $1 \% \mathrm{C}$-SWCNTs or $5 \% \mathrm{C}$-SWCNTs showed no change in the level of fibrosis, as shown in the histology images in Figure 5B, D and F, and also in the semi-quantification of the deposited collagen in Figure 6A-C. Figure 5A, C, E, G and I show histological images for the internal control of each of the treatment conditions.

CNTs improved the epithelialization process in healing wounds

Wound healing requires the regeneration of not only the dermis connective tissue layer but also the epithelial epidermis layer. 

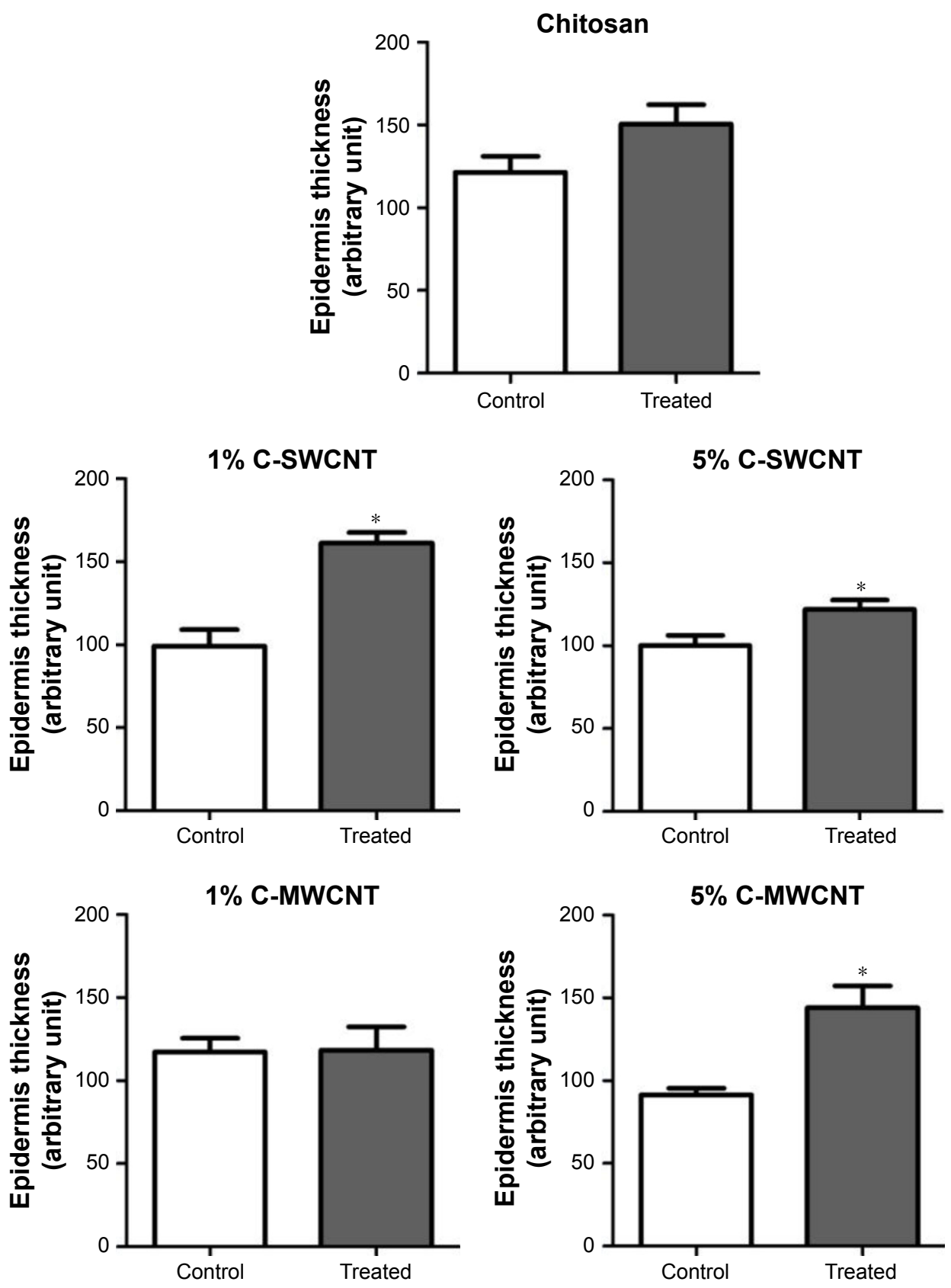

Figure 7 Both MWCNTs and SWCNTs improved the formation of the epithelial layer.

Notes: Representative random images for tissue samples stained with Masson's trichrome stain were analyzed by Image software, where the "straight line" tool was employed to arbitrarily measure the thickness of the epidermis. Values are given as means $\pm S E M, n=5$, triplicates. $* P<0.05$ compared to the control.

Abbreviations: C-SWCNT, chitosan-single-wall carbon nanotube; C-MWCNT, chitosan-multi-wall carbon nanotube.

Therefore, ImageJ was also used to the measure the thickness of the epithelium in the images of the skin tissue slides. As shown in Figure 7, samples treated with either C-SWCNTs or C-MWCNTs exhibited an increase in the thickness of the epithelium compared to the internal control, with the greatest effect being observed with 1\% C-SWCNTs and 5\% C-MWCNTs.

\section{CNTs induced collagen deposition}

The $3 \mathrm{~T} 3$ cells were cultured under different concentrations of chitosan, C-SWCNTs and C-MWCNTs. The semi-quantitative assay for the level of the deposited collagen by Sirius Red stain demonstrated that chitosan significantly increased collagen deposition compared to the control. The other treatment conditions were compared against chitosan as they contained the same concentration of chitosan in the background. C-SWCNTs significantly increased collagen deposition, by about $40 \%$, only at a concentration of $0.5 \%$. However, C-MWCNTs demonstrated a significant concentrationdependent increase in collagen deposition, with the $5 \%$ C-MWCNTs achieving an increase of about 120\% (Figure 8). 


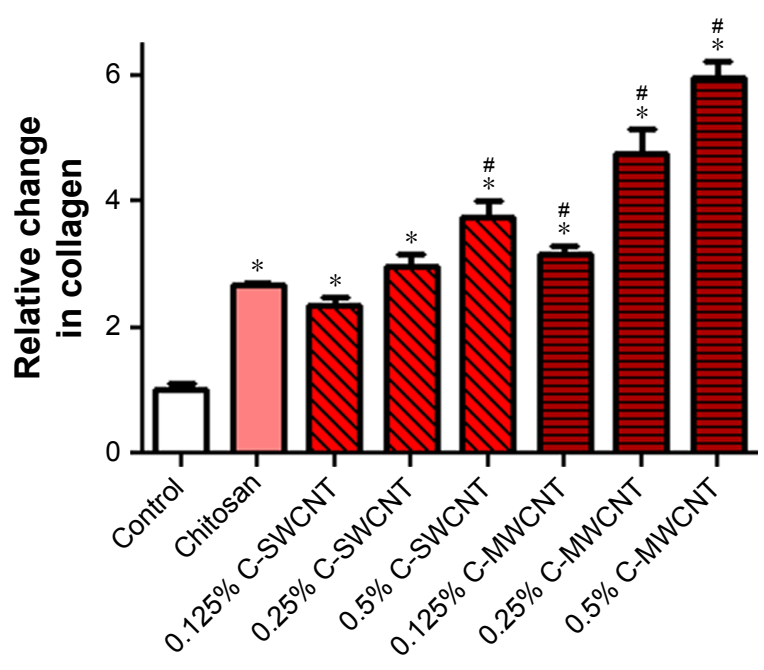

Figure 8 Both C-SWCNTs and C-MWCNTs induced collagen deposition in a concentration-dependent manner.

Notes: The potency was greater with C-MWCNT treatment. Values are given as means \pm SEM, $n=3 . * P<0.05$ compared to control; $\#<0.05$ compared to chitosan. Abbreviations: C-SWCNT, chitosan-single-wall carbon nanotube; C-MWCNT, chitosan-multi-wall carbon nanotube.

\section{Discussion}

During the past few years, CNTs have been proposed for diverse medical applications owing to their unique physical characteristics. However, pristine CNTs (CNTs without the addition of any functional group) have been shown in a number of in vitro and in vivo studies to have some toxicity at the cellular and organ levels. The addition of polar functional groups to pristine CNTs may significantly improve the dispersibility of CNTs in aqueous environments and may also greatly reduce their toxicity. ${ }^{18,19,27,28}$ In this project, C-SWCNTs and C-MWCNTs were produced by conjugating SWCNTs and MWCNTs with chitosan, which is a natural hydrophilic polymer that is well known to be biocompatible and is currently in use for the improvement of wound healing. ${ }^{4,29}$ The incorporation of C-SWCNTs and C-MWCNTs in the extracellular matrix of an ECT did not interfere with the successful formation of consistent tissue, which means that fibroblasts were able to contract and assemble the collagen fibers within the tissue, and that these complexes may not be cytotoxic in the first place. In addition, culturing fibroblasts with different concentrations of C-SWCNTs and C-MWCNTs resulted in a concentrationdependent increase in collagen deposition, which further confirms the biocompatibility of the test compounds, and a potential pro-fibrotic effect. These finding are in agreement with previous data published by Zhao et al, who also found in vitro that the presence of CNTs in chitosan/collagenbased composites promoted mouse fibroblast adhesion by producing a distinct cytoskeletal structure. ${ }^{30}$ The in vivo wound model generally demonstrated that both C-SWCNTs and C-MWCNTs improved the re-epithelialization of the healing wounds; however, they could also increase the percentage of wounds with more fibrosis, and in particular the 5\% C-MWCNTs significantly enhanced the extensiveness of this fibrosis. These observations were associated with an increase in inflammatory signs, characterized by an increase in the accumulation of inflammatory cells in the healing sites. An in vitro study by Zhang et al demonstrated that treatment of human epidermal keratinocytes with SWCNTs, functionalized with a hydrophilic group, induced the release of proinflammatory cytokines, such as IL-6 and IL-8. ${ }^{31}$ Likewise, Murray et al reported that exposure of an engineered skin tissue to SWCNTs resulted in an increase in proinflammatory cytokine release, which was accompanied by an increase in fibroblast activation and migration, collagen production and epidermal thickness. ${ }^{32}$

Inflammation plays an important role in the first stage of wound healing, where activated neutrophils secrete many lysosomal enzymes that facilitate the cleavage and elimination of damaged structural proteins of the extracellular matrix. ${ }^{33}$ In addition, the inflammatory processes recruit more macrophages to the wound bed, which secrete more inflammatory mediators and clean out the wound site, allowing the migration of activated fibroblasts to the wound site. ${ }^{34}$ The activated fibroblasts gain greater capacity to contract the wound tissue and to deposit greater amounts of glycosaminoglycans and collagen, which are essential for tissue repair. ${ }^{35}$ During the second stage of wound healing, activated fibroblasts try to compensate for the damaged extracellular matrix by depositing large amounts of collagen (fibrosis), which forms a scar. During the third stage of healing, the bulk of collagen in the scar is subjected to a long-lasting remodeling process, over a year or more, which is facilitated by metalloproteinases secreted by fibroblasts and other cells, in an attempt to strengthen the repaired tissue. However, the fibrosis is never completely resolved. ${ }^{36}$ Therefore, the concurrent increase in inflammation and collagen deposition could be interpreted as an improvement in wound healing, especially as it is also associated with improved re-epithelialization. However, further investigations are required to check whether the increase in collagen deposition would result in scarring as a side effect. In addition, CNT-containing preparations are black in color, and it was noticed that some of the healing wounds that received CNT-containing hydrogels had some small black dots, although no black color was detected in the histology samples. Therefore, we assume that the black staining by CNTs may be superficial and would not leave 
tattoo-like marks, but this needs to be confirmed in future long-term experiments.

\section{Conclusion}

In the light of the current data and the related literature, we expect that the C-MWCNT hydrogel, and perhaps to a lesser extent the C-SWCNT hydrogel, may improve wound healing. However, further follow-up investigations are required in animal species other than mice to confirm the potential therapeutic activities of these hydrogels and to elucidate possible side effects such as scarring.

\section{Acknowledgment}

This project was funded by An-Najah National University in Nablus, Palestine, through the deanship of Scientific Research under fund identification number ANNU-1718-Sc-019.

\section{Disclosure}

The authors report no conflicts of interest in this work.

\section{References}

1. Neville RF, Kayssi A, Buescher T, Stempel MS. The diabetic foot. Curr Probl Surg. 2016;53(9):408-437.

2. O’Meara S, Cullum N, Majid M, Sheldon T. Systematic reviews of wound care management: (3) antimicrobial agents for chronic wounds; (4) diabetic foot ulceration. Health Technol Assess. 2000;4(21):1-237.

3. Kittana N, Abu-Rass H, Sabra R, et al. Topical aqueous extract of Ephedra alata can improve wound healing in an animal model. Chin $J$ Traumatol. 2017;20(2):108-113.

4. Değim Z, Celebi N, Sayan H, Babül A, Erdoğan D, Take G. An investigation on skin wound healing in mice with a taurine-chitosan gel formulation. Amino Acids. 2002;22(2):187-198.

5. Kong M, Chen XG, Xing K, Park HJ. Antimicrobial properties of chitosan and mode of action: a state of the art review. Int J Food Microbiol. 2010;144(1):51-63.

6. Ueno H, Yamada H, Tanaka I, et al. Accelerating effects of chitosan for healing at early phase of experimental open wound in dogs. Biomaterials. 1999;20(15):1407-1414.

7. Mi FL, Shyu SS, Wu YB, Lee ST, Shyong JY, Huang RN. Fabrication and characterization of a sponge-like asymmetric chitosan membrane as a wound dressing. Biomaterials. 2001;22(2):165-173.

8. Biagini G, Bertani A, Muzzarelli R, et al. Wound management with N-carboxybutyl chitosan. Biomaterials. 1991;12(3):281-286.

9. Iijima S. Helical microtubules of graphitic carbon. Nature. 1991; 354(6348):56-58.

10. Cheng LC, Jiang X, Wang J, Chen C, Liu RS. Nano-bio effects: interaction of nanomaterials with cells. Nanoscale. 2013;5(9):3547.

11. Harris PJ. Carbon Nanotubes and Related Structures. Cambridge: Cambridge University Press; 1991.

12. Dresselhaus MS, Dresselhaus G, Eklund PC. Science of Fullerenes and Carbon Nanotubes: Their Properties and Applications. San Diego, CA: Academic Press; 1996.

13. Baughman RH, Zakhidov AA, de Heer WA. Carbon nanotubes - the route toward applications. Science. 2002;297(5582):787-792.

14. Yu MF, Files BS, Arepalli S, Ruoff RS. Tensile loading of ropes of single wall carbon nanotubes and their mechanical properties. Phys Rev Lett. 2000;84(24):5552.
15. Meo M, Rossi M. Prediction of Young's modulus of single wall carbon nanotubes by molecular-mechanics based finite element modelling. Compos Sci Technol. 2006;66(11-12):1597-1605.

16. Rao CN, Satishkumar BC, Govindaraj A, Nath M. Nanotubes. Chemphyschem. 2001;2(2):78-105.

17. Reich S, Thomsen C, Maultzsch J. Carbon Nanotubes: Basic Concepts and Physical Properties. Weinheim: Wiley; 2004.

18. Ema M, Gamo M, Honda K. A review of toxicity studies of singlewalled carbon nanotubes in laboratory animals. Regul Toxicol Pharmacol. 2016;74:42-63.

19. Madani SY, Mandel A, Seifalian AM. A concise review of carbon nanotube's toxicology. Nano Rev. 2013;4:21521.

20. Liu Z, Robinson JT, Tabakman SM, Yang K, Dai H. Carbon materials for drug delivery \& cancer therapy. Mater Today. 2011;14(7-8): 316-323.

21. Zhang W, Zhang Z, Zhang Y. The application of carbon nanotubes in target drug delivery systems for cancer therapies. Nanoscale Res Lett. 2011;6:555.

22. Assali M, Zaid AN, Kittana N, Hamad D, Amer J. Covalent functionalization of SWCNT with combretastatin A4 for cancer therapy. Nanotechnology. 2018;29(24):245101.

23. Furuike T, Komoto D, Hashimoto H, Tamura H. Preparation of chitosan hydrogel and its solubility in organic acids. Int J Biol Macromol. 2017;104(Pt B):1620-1625.

24. Tiburcy M, Meyer T, Soong PL, Zimmermann WH. Collagen-based engineered heart muscle. Methods Mol Biol. 2014;1181:167-176.

25. Chen Y, Qi Y, Xu C-B. A convenient method for quantifying collagen fibers in atherosclerotic lesions by ImageJ software. Int J Clin Exp Med. 2017;10(10):14904-14910.

26. Tullberg-Reinert H, Jundt G. In situ measurement of collagen synthesis by human bone cells with a sirius red-based colorimetric microassay: effects of transforming growth factor beta2 and ascorbic acid 2-phosphate. Histochem Cell Biol. 1999;112(4):271-276.

27. Zhang T, Tang M, Zhang S, et al. Systemic and immunotoxicity of pristine and PEGylated multi-walled carbon nanotubes in an intravenous 28 days repeated dose toxicity study. Int J Nanomedicine. 2017;12: 1539-1554.

28. Zhang T, Tang M, Kong L, et al. Comparison of cytotoxic and inflammatory responses of pristine and functionalized multi-walled carbon nanotubes in RAW 264.7 mouse macrophages. J Hazard Mater. 2012: 219203-220212.

29. Dreifke MB, Jayasuriya AA, Jayasuriya AC. Current wound healing procedures and potential care. Mater Sci Eng C Mater Biol Appl. 2015; 48:651-662.

30. Zhao W, Yu W, Zheng J, Wang Y, Zhang Z, Zhang D. Effects of carbon nanotubes in a chitosan/collagen-based composite on mouse fibroblast cell proliferation. Cell Mol Neurobiol. 2014;34(1):43-50.

31. Zhang LW, Zeng L, Barron AR, Monteiro-Riviere NA. Biological interactions of functionalized single-wall carbon nanotubes in human epidermal keratinocytes. Int J Toxicol. 2007;26(2):103-113.

32. Murray AR, Kisin E, Leonard SS, et al. Oxidative stress and inflammatory response in dermal toxicity of single-walled carbon nanotubes. Toxicology. 2009;257(3):161-171.

33. Simpson DM, Ross R. The neutrophilic leukocyte in wound repair a study with antineutrophil serum. J Clin Invest. 1972;51(8):2009-2023.

34. Diegelmann RF, Evans MC. Wound healing: an overview of acute, fibrotic and delayed healing. Front Biosci. 2004;9:283-289.

35. Ilaria Tocco BZ, Bassetto F, Vindigni V. Nanotechnology-Based Therapies for Skin Wound Regeneration. Journal of Nanomaterials. 2012;714134.

36. Gurtner GC, Werner S, Barrandon Y, Longaker MT. Wound repair and regeneration. Nature. 2008;453(7193):314-321. 


\section{Publish your work in this journal}

The International Journal of Nanomedicine is an international, peerreviewed journal focusing on the application of nanotechnology in diagnostics, therapeutics, and drug delivery systems throughout the biomedical field. This journal is indexed on PubMed Central, MedLine, CAS, SciSearch $\AA$, Current Contents $\AA /$ Clinical Medicine,

Journal Citation Reports/Science Edition, EMBase, Scopus and the Elsevier Bibliographic databases. The manuscript management system is completely online and includes a very quick and fair peer-review system, which is all easy to use. Visit http://www.dovepress.com/ testimonials.php to read real quotes from published authors.

Submit your manuscript here: http://www.dovepress.com/international-journal-of-nanomedicine-journal 